\title{
Amblyopic Deficit Beyond the Fovea: Delayed and Variable Single-Trial ERP Response Latencies, but Unaltered Amplitudes
}

\author{
Éva M. Bankó, ${ }^{1}$ Judit Körtvélyes, ${ }^{2}$ János Németh, ${ }^{2}$ and Zoltán Vidnyánszky ${ }^{1,3}$ \\ ${ }^{1}$ Research Centre for Natural Sciences, Hungarian Academy of Sciences, Budapest, Hungary \\ ${ }^{2}$ Department of Ophthalmology, Semmelweis University, Budapest, Hungary \\ ${ }^{3}$ Department of Cognitive Science, Budapest University of Technology and Economics, Budapest, Hungary
}

Correspondence: Éva M. Bankó, Magyar tudósok körútja 2., 1117 , Budapest, Hungary;

banko.eva@ttk.mta.hu.

Submitted: October 30, 2013

Accepted: January 7, 2014

Citation: Bankó ÉM, Körtvélyes J, Németh J, Vidnyánszky Z. Amblyopic deficit beyond the fovea: delayed and variable single-trial ERP response latencies, but unaltered amplitudes. Invest Ophthalmol Vis Sci. 2014;55:1109-1117. DOI:10.1167/ iovs.13-13507
Purpose. Amblyopia was first described as a deficit of central vision. However, it has long been debated whether this dysfunction is limited to the fovea or whether extrafoveal vision is also affected, as studies concerning the latter are equivocal. The purpose of the study was to resolve this issue.

Methods. We investigated the amblyopic effect on event-related potentials (ERPs) with foveal and perifoveal stimuli, either matched in size based on cortical magnification or presented as large annular stimuli. In two separate experiments we measured ERPs on amblyopic patients and control subjects using face images. Latency and amplitude of averaged ERPs and their single-trial distributions were analyzed.

REsults. When the fovea was stimulated, latency and amplitude of the early averaged ERP components increased and were reduced, respectively, in the amblyopic compared with the fellow eye. Importantly, perifoveal stimulation also elicited similar amblyopic deficits, which were clearly significant in the case of using cortical magnification scaled stimuli. However, single-trial peak analysis revealed that foveal and perifoveal effects differed in nature: Peak amplitudes were reduced only in foveal stimulation, while latencies were delayed and jittered at both the fovea and perifovea. Event-related potentials obtained from fellow eyes were not significantly different from those of normal observers.

Conclusions. Our findings revealed the existence of amblyopic deficits at the perifovea when the stimulated cortical area was matched in size to that of foveal stimulation. These deficits manifested themselves only in the temporal structure of the responses, unlike foveal deficits, which affected both component amplitude and latency.

Keywords: single-trial peak detection, visual cortex, cortical magnification, P1, ERP

\begin{abstract}
A mblyopia is a visual disorder of decreased acuity and Aabnormal visual function caused by an anomalous early visual experience. Traditionally, it has been regarded as a disorder limited to the central retina, ${ }^{1}$ even though there exist studies that question this notion. ${ }^{2,3}$ As the results collected over some four decades are equivocal, no consensus has so far been reached regarding how the peripheral visual field is affected in amblyopia. Today only strabismic amblyopia is considered a deficit primarily of central vision, as early psychophysical investigations found that contrast detection threshold, ${ }^{4}$ acuity, ${ }^{5-7}$ and binocular interactions ${ }^{8}$ are similar between the two eyes from eccentricities of $20^{\circ}$ on. This is in agreement with macaque single unit recording ${ }^{9}$ and human functional magnetic resonance imaging studies $^{10}$ that also found no peripheral interocular differences in strabismic amblyopia. In contrast, other studies investigating both strabismic and anisometropic amblyopes have shown decreased sensitivity of the amblyopic eye in the periphery for motion detection and discrimination ${ }^{3}$ and contrast detection ${ }^{2}$ in the eccentricity range of $10^{\circ}$ to $30^{\circ}$. The extent of the amblyopic loss in the periphery in both experiments was related to the degree of foveal loss rather than the type of amblyopia.
\end{abstract}

Copyright 2014 The Association for Research in Vision and Ophthalmology, Inc www.iovs.org | ISSN: 1552-5783
Electrophysiological studies have also led to different results concerning the periphery in amblyopia. Full-field patternreversal visual evoked potential (VEP) studies ${ }^{11,12}$ support the dominantly central deficit in amblyopia based on the lack of interocular difference with the use of large check sizes $\left(>60^{\prime}\right)$, where responses are thought to predominantly arise from neurons processing the periphery of the visual field. ${ }^{11,13-15}$ Stimulation of the amblyopic eye with small check sizes $\left(<30^{\prime}\right)$, on the other hand, which preferentially activates the foveal area ${ }^{11,13-15}$ as it elicits measurable VEP responses up to only $2^{\circ}$ to $4^{\circ}$ eccentricity, ${ }^{13}$ yields drastically reduced and delayed VEP responses. Similar divergence is obtained in studies using small central and large annular stimuli for the stimulation of the fovea and perifovea, respectively. ${ }^{10,16}$ As opposed to full-field VEP, the multifocal VEP (mfVEP) technique is capable of directly investigating peripheral processing by stimulating the visual field at different eccentricities. These studies, on the other hand, tend to find amplitude and latency differences at the perifoveal region as well as at the fovea, even though they are smaller in size. ${ }^{17-19}$

A few issues, if considered, might help to reconcile the different conclusions of these studies. One is the effect of 
decreased acuity on the event-related potentials (ERPs). It has been shown that induced refractive error causes amplitude reduction of VEP components, which is most pronounced for stimuli with higher spatial frequency (e.g., checks of $5^{\prime}$ to $40^{\prime}$ of arc) ${ }^{20,21}$ Increasing the refractive error in either direction not only reduces VEP amplitudes for the different check sizes but also shifts the peak sensitivity to larger check sizes. Thus, large refractive error can mask interocular amplitude differences for small spatial frequencies. Another point is the difference between the methodologies in the area of peripheral stimulation. The stimulation field size in an mfVEP stimulus is scaled with eccentricity ${ }^{22}$ using the cortical magnification formula computed by Horton and Hoyt, ${ }^{23}$ keeping the number of reversing checks constant across stimulation subfields ${ }^{24}$; in contrast, the typical clinical pattern-reversal VEP applications use a homogeneous large stimulation field minimally $15^{\circ}$ in diameter, ${ }^{14}$ which does not scale with check size, hence ignoring cortical magnification. The same holds true for large annular stimuli that are also frequently used to stimulate the perifovea. ${ }^{10,16}$ Thus, with the use of full-field VEPs or annular central stimuli ${ }^{10}$ to investigate perifoveal or peripheral processing, many more neurons are activated in perifoveal compared to foveal stimulation. Due to the extensive summation of activity evoked by a large number of neurons throughout the whole periphery, ${ }^{2}$ small extrafoveal deficits could in principle fail to reach significance, hence go undetected.

Therefore, our primary goal in this study was to investigate cortical processing of the amblyopic eye outside the foveal area by scaling the stimulus size, thus keeping the stimulated area of the visual cortex constant at different eccentricities. In a separate experiment we also investigated this issue using large annular stimuli, which ignore cortical magnification. We hypothesized that if amblyopic deficits exist outside the fovea, controlling for cortical magnification could reveal interocular differences that might otherwise be masked by large full-field stimulation. ${ }^{2,10}$ We utilized single-trial peak detection to uncover the nature of the deficits found. To rule out the possibility that the amblyopic effects are simply due to the loss of higher spatial frequencies as a result of decreased acuity-a phenomenon inevitably occurring during amblyopic viewing and known to affect ERP components ${ }^{20,21}$-we measured ERPs to low-pass-filtered stimuli as well.

\section{Materials AND Methods}

\section{Subjects}

Fifteen (Experiment 1; mean age \pm SD: $28 \pm 7$ years) and 14 amblyopic patients (Experiment 2; mean age \pm SD: $37 \pm 10$ years) gave their informed and written consent to participate in the study, which was approved by the ethics committee of Semmelweis University and was in accordance with the Declaration of Helsinki. All subjects were examined by an ophthalmologist and fitted with optimal correction. Inclusion criteria for amblyopic patients were the following: best corrected visual acuity of the fellow eye of 20/20 or better, best corrected visual acuity of the amblyopic eye in the range of $20 / 25$ to $20 / 200$ with no ocular organic abnormalities present (except for refraction error or squint). Supplementary Table S1 details their medical parameters. (Experiment 2 was also conducted on 14 healthy control subjects; for full description and results see Supplementary Material.)

\section{Visual Stimuli and Procedures}

In Experiment 1, participants viewed face images tilted $5^{\circ}$ to the right or left from the vertical meridian. Six black-and-white face photographs (three male and three female) were taken from our face database and cropped to $254 \times 254$ pixels, covered with a circular mask to eliminate external facial features, and equated for luminance and contrast. The faces were either displayed without further manipulation, containing a broad spatial spectrum $(\mathrm{Br})$, or low-pass filtered at three cycles/image (Lo) using the ImageJ tool (http://rsbweb.nih. gov/ij/, in the public domain) ${ }^{25}$ (Supplementary Fig. S1A). The high-frequency cutoff for the low-pass filter was chosen to exclude spatial frequencies higher than 1.5 cycles per degree (cpd) in the case of foveal stimuli $\left(2^{\circ}\right)$, as contrast sensitivity of the amblyopic eye at $1.5 \mathrm{cpd}$ assessed by the Sine Wave Contrast Test was found to be in the normal range for all of our subjects (Supplementary Fig. S1C). We chose faces for stimuli as opposed to the more conventional checkerboard or sine wave grating/Gabor patch stimuli for several reasons, as follows. They are natural, ecologically valid stimuli better suited to investigating processing deficits that limit amblyopic patients in real life; we could control their spatial frequency content just as well as with Gabor patches, by filtering out unwanted frequencies; and we had prior knowledge about the validity of our single-trial peak detection approach on the ERPs evoked by faces-they tend to be big, thus having a good signal-to-noise ratio, compared with ERPs evoked by simple stimuli. Even though it has been previously shown that there is a face-specific processing deficit in amblyopia, ${ }^{26,27}$ the amblyopic deficit in early neural processing, as reflected in the $\mathrm{P} 1$ component, should not be significantly affected by the stimulus used.

On half of the trials, faces were presented centrally subtending $2^{\circ}$ (corresponding to the size of the fovea), while on the other half, they were presented at $5^{\circ}$ eccentricity along either the upper or the lower vertical meridian. The vertical meridian was used to avoid the known nasotemporal asymmetries of strabismic amblyopes, ${ }^{7,18}$ which would have increased the within-subject variance had the perifoveal stimuli been presented along the horizontal meridian. To control for the decrease in the retinal and cortical representation of the visual field toward the periphery, the $2^{\circ}$ images were scaled with the cortical magnification factor and presented at the size of $4.7^{\circ}$ in the perifoveal condition (covering $2.65^{\circ}$ to $7.35^{\circ}$ eccentricity). Image size was determined with the formula $M_{\text {linear }}=\frac{A}{E+E_{2}}$ provided by Horton and Hoyt ${ }^{23}$ using $A=29.2$ $\mathrm{mm} E_{2}=3.67^{\circ}$ as calculated for human V1. ${ }^{28}$

Each trial started with a cue, a brief change $(100 \mathrm{~ms})$ in the color of the fixation dot followed by the face stimulus for 250 ms with a fixed stimulus onset asynchrony of $1350 \mathrm{~ms}$. A response window of 2 seconds was given, which terminated when the subjects responded. The patients' task was to judge the orientation of the face images and indicate a leftward or rightward tilt with the left or right mouse button, respectively. Trials were separated by a random intertrial interval of 800 to $1200 \mathrm{~ms}$. A fixation dot was present throughout the entire block, and subjects were instructed to maintain fixation throughout the experiment. Stimuli were presented on a uniform gray background. Broad spatial spectrum and Lo face stimuli were presented with equal probability within a block in random order, as were foveal and perifoveal presentations of these stimuli. Viewing was monocular, alternating between blocks, while the other eye was patched. Each participant completed four runs for each eye, yielding 108 trials in total for each stimulus type per eye (for a total of 864 trials). Stimulus presentation was controlled by MATLAB 7.1 (The MathWorks, Inc., Natick, MA) using the Cogent 2000 toolbox (http://www. vislab.ucl.ac.uk/cogent_2000.php, in the public domain); stimuli were presented on a 26-inch LG IPS panel LCD monitor (LG Electronics, Inc., Yeouido-dong, Seoul, South Korea) with 
large viewing angles at a refresh rate of $60 \mathrm{~Hz}$ and were viewed from $56 \mathrm{~cm}$.

In Experiment 2 we investigated the effect of large-field perifoveal stimulation. Subjects categorized faces and hands presented in two sizes: foveal $\left(2^{\circ}\right)$ and perifoveal $\left(15^{\circ}\right.$ with the central $1.5^{\circ}$ masked, yielding an annulus subtending $0.75^{\circ}$ to $7.5^{\circ}$ eccentricity). The stimulation protocol was similar to the one described for Experiment 1 with minor differences in timing. In the present study, only face trials were analyzed. (For full methodological description and detailed results, see Supplementary Material.)

\section{Electrophysiological Acquisition and Processing}

A detailed technical description of acquisition and preprocessing was presented by Bankó et al. ${ }^{27}$ Briefly, electroencephalographic (EEG) data were acquired using a BrainAmp MR (Brain Products $\mathrm{GmbH}$, Munich, Germany) amplifier from $60 \mathrm{Ag} / \mathrm{AgCl}$ scalp electrodes mounted on an EasyCap (Easycap $\mathrm{GmbH}$, Herrsching-Breitbrunn, Germany) with four additional periocular electrodes for recording the electrooculogram. All input impedance was kept below $5 \mathrm{k} \Omega$. Channels were referenced to joint earlobes online with the nasion as ground and were rereferenced offline using a Laplacian transform on spherical spline interpolated data to generate scalp current density (SCD) waveforms. This was done to eliminate contamination of saccadic potentials and to make the data better suited for single-trial peak detection (for more information see Bankó et al. ${ }^{27}$ ). Data were band-pass filtered from 0.1 to $30 \mathrm{~Hz}$ including a $50-\mathrm{Hz}$ notch filter, segmented ( -200 to $600 \mathrm{~ms}$ relative to stimulus), artifact rejected, and baseline corrected. Data processing was done using BrainVision Analyzer (Brain Products $\mathrm{GmbH}$ ).

\section{Statistical Analysis}

Accuracy, calculated as percent correct responses, and reaction time were evaluated as behavioral measures. P1 and N170 component peaks were detected and analyzed on electrodes showing maximum deviation relative to baseline in the group average in the expected time period corresponding to the ERP peaks (PO7, PO9, P7, and P9 and PO8, PO10, P8, and P10 for left and right clusters, respectively, for both components). In the case of averaged ERPs, peak latency was determined on the left and right clusters separately, while mean peak amplitudes were measured over the individual electrodes of the clusters in a 10-ms window. For single-trial peak analysis, peaks were detected on each trial for each electrode as maximum and minimum activity for P1 and N170, respectively, in an 80-ms time window centered on the individual peak latency of the respective component measured on the averaged ERPs. The amplitude and corresponding time of the local extremes were taken as the amplitude and latency of the component on a given trial. Single-trial amplitude and latency values were pooled from electrodes on each side; the distribution of the values was characterized by calculating the median and the interquartile range (IQR), which is a measure of spread and is computed as the difference of the upper and lower quartile of the data and thus describes the middle $50 \%$ of the data values. ${ }^{27}$

Foveal and perifoveal data were analyzed separately by repeated-measures ANOVAs with within-subject factors of eye (fellow eye [FE] versus amblyopic eye $[\mathrm{AE}])$ and filtering $(\mathrm{Br}$ versus $L O$ ) for behavioral measures; eye, filtering, side (left [L] versus right $[\mathrm{R}]$ ), and electrode for averaged ERP amplitude; and eye, filtering, and side for averaged ERP latency and singletrial electrophysiological measures, as these measures were pooled across electrodes for obtaining more reliable estimates of the central tendency and dispersion of the distributions. Tukey honestly significant difference tests were used for post hoc comparisons. Homogeneity of variances was tested using Bartlett's test for equal variances; and in case this assumption was not met due to the higher variance of measurements from the $\mathrm{AE}$, values were rank transformed before being entered into the statistical test, which is noted by superscript $r$ for rank ANOVA next to $F$ values when statistical results are detailed. As many separate ANOVAs were conducted for analyzing the behavioral and electrophysiological data, significance level was set to $P=0.013(\sim 0.05 / 4$ : four main comparisons of two positions $\times$ two independent measures) to control for the inflated type I error rate as a result of multiple comparisons. The significance level for fixation measurements was kept at $P$ $=0.05$. We also conducted correlation analyses between the amblyopic effect on behavioral and electrophysiological measures using Spearman rank correlation. The interocular difference of all variables was taken as the index of the amblyopic effect.

\section{Analysis of Eye-Tracking Data}

We tracked the gaze direction of all subjects using the iViewX Hi-Speed tracking column (SMI GmbH, Teltow, Germany) while they performed the EEG experiment. However, we were able to record usable eye movement data for only nine patients due to the strong reflection of glasses that many were wearing. Trials were binned based on the viewing eye, stimulus type, and stimulus position; then, for each eye position measurement (i.e., a pair of $[x, y]$ coordinates), geometrical distance from the fixation point was calculated. The median distance of each of the eight stimulation conditions was used as a measure of fixation stability in each subject, higher distance values meaning less stable fixation. Analysis was carried out using three-way repeated-measures ANOVA with eye (FE versus AE), filtering ( $\mathrm{Br}$ versus $\mathrm{Lo}$ ), and position (fovea versus perifovea) as within-subject factors.

\section{Results}

\section{Behavioral Results}

The patients performed the task with near ceiling accuracy. Nevertheless, amblyopic performance was slightly but significantly worse than that of the fellow eye at the fovea, while this difference remained only a trend at the perifovea (Fig. 1A). Reaction times were significantly longer for the amblyopic eye in both stimulus conditions (Fig. 1B). Furthermore, low-pass filtering the faces affected accuracy and reaction time similarly to the amblyopic effect (statistical analysis can be found in the Supplementary Material).

The results of the eye-tracking analysis revealed that in agreement with previous findings, ${ }^{19,27,29-31}$ the ability of the amblyopic eye to fixate the central fixation mark was poor compared with that of the fellow eye (Fig. 1C; eye: $F_{(1,8)}=$ 9.39, $P=0.015)$. Importantly, however, this difference was constant across the visual field. Overall fixation stability was not affected by either stimulus position (position: $F_{(1,8)}=0.04$, $P=0.85$; eye $\times$ position: $F_{(1,8)}=0.85, P=0.38$ ) or low-pass filtering (filtering: $F_{(1,8)}=1.79, P=0.22$ ).

There were no systematic relationships between the amblyopic effect in any of the behavioral measures and the interocular difference in electrophysiological measures. A possible explanation for the lack of correlations is that the task was too easy to warrant expectation of a substantial modulation in the behavioral results. 


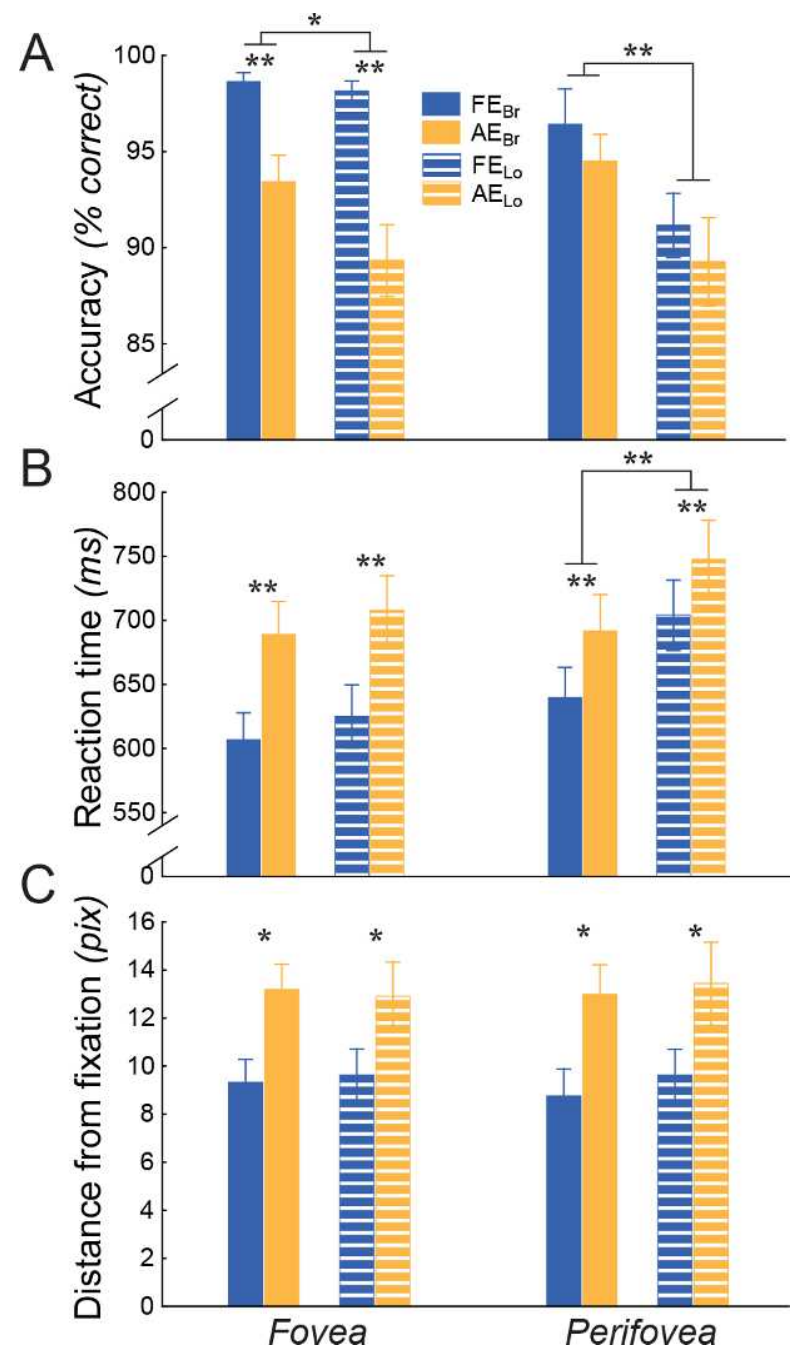

Figure 1. Behavioral results: accuracy (A), reaction times (B), and fixation stability (C). Results obtained from the AE are shown in yellow, while results from the $\mathrm{FE}$ are blue. $\mathrm{Br}$, faces with broad spatial frequency content, solid columns; Lo, low-pass-filtered face stimuli, striped columns; asterisks denote significant differences $\left({ }^{*} P<0.013\right.$, ** $P<0.001 ; n=15)$.

\section{Averaged ERPs Show Amblyopic Deficit at Both the Fovea and Perifovea}

Foveal Stimulation. The results revealed strong amblyopic effects on the amplitude and latency of the P1 and N170 components of the averaged ERPs in the case of foveal stimuli (Fig. 2A, Supplementary Fig. S2A), which were in accordance with previous findings. ${ }^{11,27,32-34}$ Viewing with the amblyopic eye led to reduced amplitudes and delayed latencies compared with the fellow eye for both ERP components (for statistics see Table 1). These effects were similar for $B r$ and $L o$ stimuli, as no significant eye $\times$ filtering interactions were found. Interestingly, the only effect that low-pass filtering had on the averaged ERPs was a decrease in the averaged ERP amplitudes of the P1 component in both eyes.

Perifoveal Stimulation. Stimulation of the perifoveal region while cortical magnification was controlled yielded clear amblyopic deficits on the amplitude and latency of both ERP components similar to those found in foveal stimulation (Fig. 2B, Supplementary Fig. S2B, Table 1): Averaged component amplitudes were reduced while latencies increased in the amblyopic eye compared with the fellow eye for both ERP components. Here too, these effects were present for both $\mathrm{Br}$ and $L o$ stimuli with the exception of P1 latency, where only $\mathrm{Br}$ stimuli differed between eyes while the trend for $L O$ stimuli did not reach significance. Low-pass filtering the perifoveal images affected neither the amplitude nor the latency of ERP components.

Large-Field Perifoveal Stimulation. Importantly, with the use of large annular stimuli to activate the perifovea in Experiment 2, the amblyopic effects on amplitude and latency of the P1 component failed to reach significance, even though a trend similar to the findings obtained with foveal images was present. In the case of N170, there was slight but significant increase in component latency and a nonsignificant trend of amplitude reduction under amblyopic viewing (Fig. 2C; statistical evaluation and more details can be found in the Supplementary Material).

With all the observations taken together, amblyopia affects the component amplitude and latency of averaged ERPs under both foveal and perifoveal stimulation; but for the latter to be statistically evident it is advisable to keep the area of the activated cortex equal as stimulation is moved toward the periphery of the visual field. Importantly, however, amblyopic effects at the perifovea were small in contrast to those with foveal stimulation, which were statistically significant for most measures (Table 1).

\section{Single-Trial Amplitude Amblyopic Deficit Is Restricted to the Fovea}

We were interested in whether this magnitude difference between fovea and perifovea simply reflected a quantitative decrease in the deficits toward the periphery, as has been suggested, ${ }^{4,7,8}$ or whether qualitative changes may underlie amblyopic processing deficits at the periphery compared with the fovea. However, the results from the averaged ERP peak analysis are insufficient to pin down the nature of the amblyopic effects due to contamination of the observed amplitude by the elevated trial-to-trial ERP latency jitter in the amblyopic compared with the fellow eye, ${ }^{27}$ which is a result of impaired temporal structure of neural responses elicited by the stimulation of the amblyopic eye. ${ }^{35-37}$ Therefore, we performed a single-trial peak analysis on the responses obtained from faces with broad spatial frequency content by detecting peaks on each trial and evaluating component amplitude and latency distributions. This enabled us to separate the contribution of changes in single-trial amplitude and latency to the amblyopic effects observed at the fovea and perifovea.

Foveal Stimulation. In the case of foveal stimulation, single-trial response amplitudes were reduced significantly in the amblyopic compared with the fellow eye for both ERP components, which was evident in a shift of the amplitude distributions toward smaller values as indicated by a decrease in their medians (Figs. 3A, 4A; see Table 2 for statistics). This drop, however, was significant only on the right side in the case of P1 while present over both hemispheres but more pronounced on the right side for N170. Dispersion of the amplitude values coming from the amblyopic eye was similar to that for the fellow eye; thus, the spread of component amplitude distributions was not altered by amblyopic viewing.

Perifoveal Stimulation. Importantly, however, amplitude distributions corresponding to peripheral stimulation, in contrast to foveal stimulation, were not affected by amblyopia. Distributions, as characterized by their median and spread, were similar between eyes for both components (Figs. 3B, 4B, Table 2). 


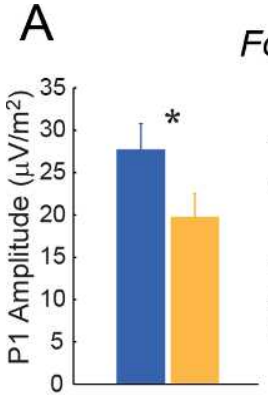

Fovea
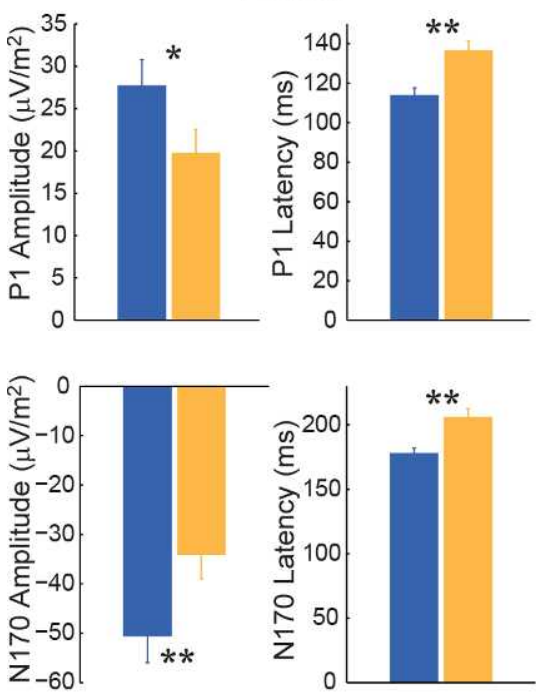

B

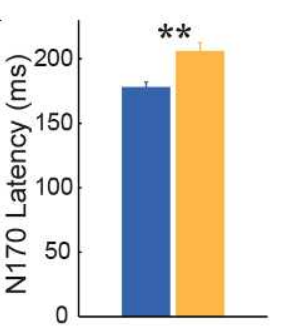

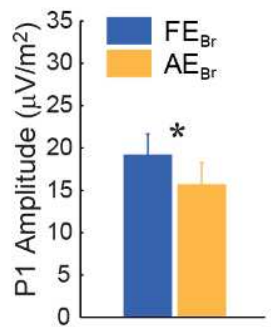

Perifovea
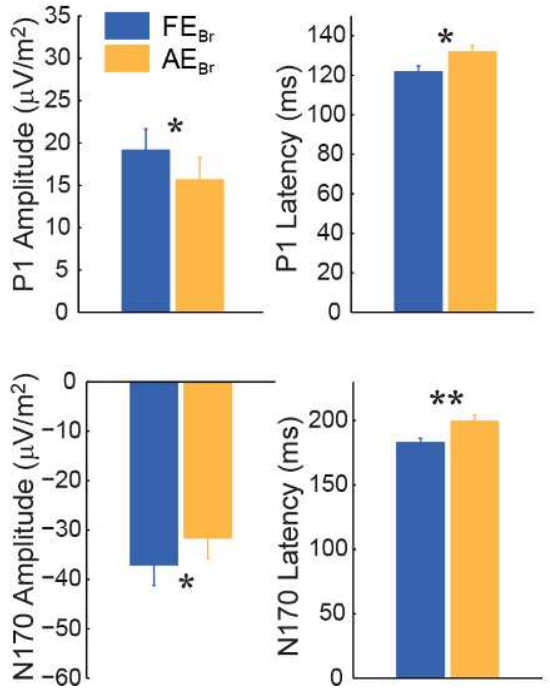

C Large-field perifovea
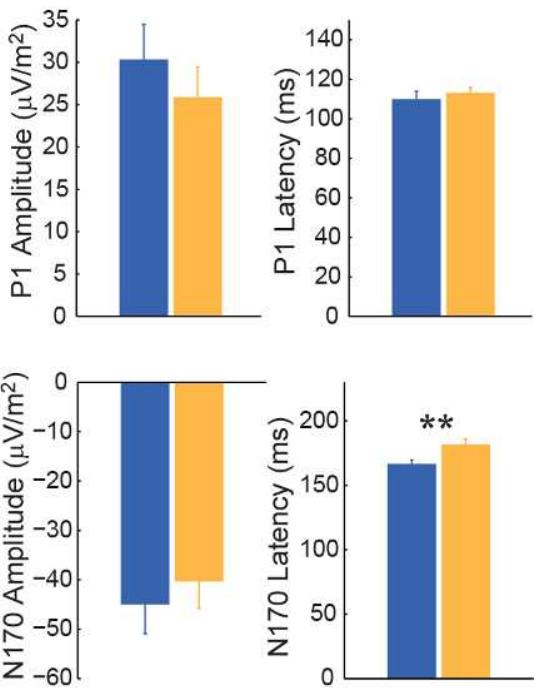

FIGURE 2. Amplitude and latency of averaged event-related potentials of amblyopic subjects for foveal (A) and perifoveal (B) presentation. Stimuli were matched in size according to the cortical magnification factor $(n=15)$. (C) Statistics for large-field perifoveal stimuli from Experiment 2 are shown for comparison. Trends similar to those in (A) and (B) can be seen, but large-field stimulation masks the amblyopic deficits, decreasing the sensitivity to detect them (see Supplementary Material; $n=14$ ). AE, yellow; FE, blue; asterisks denote significant differences $\left({ }^{*} P<0.013\right.$, ${ }^{* *} P<$ $0.001)$.

\section{Amblyopic Latency Distributions Display Both Foveal and Perifoveal Deficit}

Foveal Stimulation. P1 and N170 latency distributions were affected by amblyopic viewing, which led to a shift toward longer latencies (i.e., elevated medians) and to an increase in trial-to-trial latency jitter (i.e., larger spreads) (Figs. 3A, 4A, Table 2). These effects were similar across hemispheres except in the case of the N170 latency medians, where the right hemisphere displayed a bigger amblyopic delay compared to the left hemisphere (median difference: 38 vs. $21 \mathrm{~ms}$ ), which remained a nonsignificant trend. This replicates our previous results showing a selective processing deficit for faces in amblyopia. ${ }^{27}$

Perifoveal Stimulation. When faces were presented at the perifovea, P1 and N170 latency distributions coming from the amblyopic eye displayed a pattern similar to that for foveal stimulation: increased medians and spreads compared with the fellow eye (Figs. 3B, 4B, Table 2). Latency medians were larger over both hemispheres, while the spread of latency distributions displayed a significant difference between amblyopic and normal viewing only over the right hemisphere.

When the data are taken together, averaged component amplitude reduction at the fovea stems from a mixture of single-trial amplitude decrease and the elevation of trial-to-trial latency jitter. In contrast, at the perifovea it is predominantly the result of increased trial-to-trial component jitter. Thus, the apparent averaged amplitude reduction in the latter case arises from averaging and is not due to a decrease in response magnitude. Conversely, the amblyopic delay in component latencies is present for both foveal and perifoveal stimulation, indicating a true neural deficit outside the fovea.

TABLE 1. Amplitude and Latency Statistics for the Averaged ERP Responses

\begin{tabular}{|c|c|c|c|c|}
\hline & \multicolumn{2}{|c|}{ Fovea } & \multicolumn{2}{|c|}{ Perifovea } \\
\hline & Amplitude & Latency & Amplitude & Latency \\
\hline \multicolumn{5}{|l|}{$P 1^{*}$} \\
\hline Eye & $F_{(1,14)}=14.18, P=0.0021$ & $F_{(1,14)}^{r}=60.21, P<0.0001$ & $F_{(1,14)}=11.11, P=0.0049$ & $F_{(1,14)}=15.67, P=0.0014$ \\
\hline Filtering & $F_{(1,14)}=23.42, P=0.0003$ & $F_{(1,14)}^{r_{14}}<0.001, P=0.98$ & $F_{(1,14)}=1.50, P=0.24$ & $F_{(1,14)}=1.08, P=0.32$ \\
\hline Eye $\times$ filtering & $F_{(1,14)}=0.93, P=0.35$ & $F_{(1,14)}^{r}=0.05, P=0.83$ & $F_{(1,14)}=2.97, P=0.11$ & $\begin{array}{c}\boldsymbol{F}_{(\mathbf{1}, 14)}=9.08, P=0.0093 \\
\mathbf{F E}_{\boldsymbol{B} \boldsymbol{r}} \text { vs. } \mathrm{AE}_{\boldsymbol{B} \boldsymbol{r}}, \boldsymbol{P}=\mathbf{0 . 0 0 0 2} \\
\mathrm{FE}_{L o} \text { vs. } \mathrm{AE}_{L o}, P=0.021\end{array}$ \\
\hline \multicolumn{5}{|l|}{ N1† } \\
\hline Eye & $F_{(1,14)}=23.28, P=0.0003$ & $F_{(1,14)}^{r}=49.71, P<0.0001$ & $F_{(1,14)}=16.49, P=0.0012$ & $F_{(1,14)}=19.73, P=0.0005$ \\
\hline Filtering & $F_{(1,14)}=2.05, P=0.17$ & $F_{(1,14)}^{r}=1.17, P=0.29$ & $F_{(1,14)}=2.45, P=0.14$ & $F_{(1,14)}=0.59, P=0.46$ \\
\hline Eye $\times$ filtering & $F_{(1,14)}=3.77, P=0.073$ & $F_{(1,14)}^{r}=0.01, P=0.93$ & $F_{(1,14)}=0.03, P=0.86$ & $F_{(1,14)}=0.38, P=0.54$ \\
\hline
\end{tabular}

Significant effects are in boldface. ANOVA conducted on ranked data is denoted by the superscript $r$.

${ }^{*}$ Eye $\times$ position: $F_{(1,14)}=5.58, P=0.033$ and $\boldsymbol{F}_{(1,14)}=\mathbf{1 6 . 4 5}, \boldsymbol{P}=\mathbf{0 . 0 0 1 1}$ for amplitude and latency, respectively.

† Eye $\times$ position: $\boldsymbol{F}_{(1,14)}=\mathbf{1 4 . 1 8}, \boldsymbol{P}=\mathbf{0 . 0 0 2 1}$ and $\boldsymbol{F}_{(1,14)}=\mathbf{1 0 . 0 3}, \boldsymbol{P}=\mathbf{0 . 0 0 6 9}$ for amplitude and latency, respectively. 

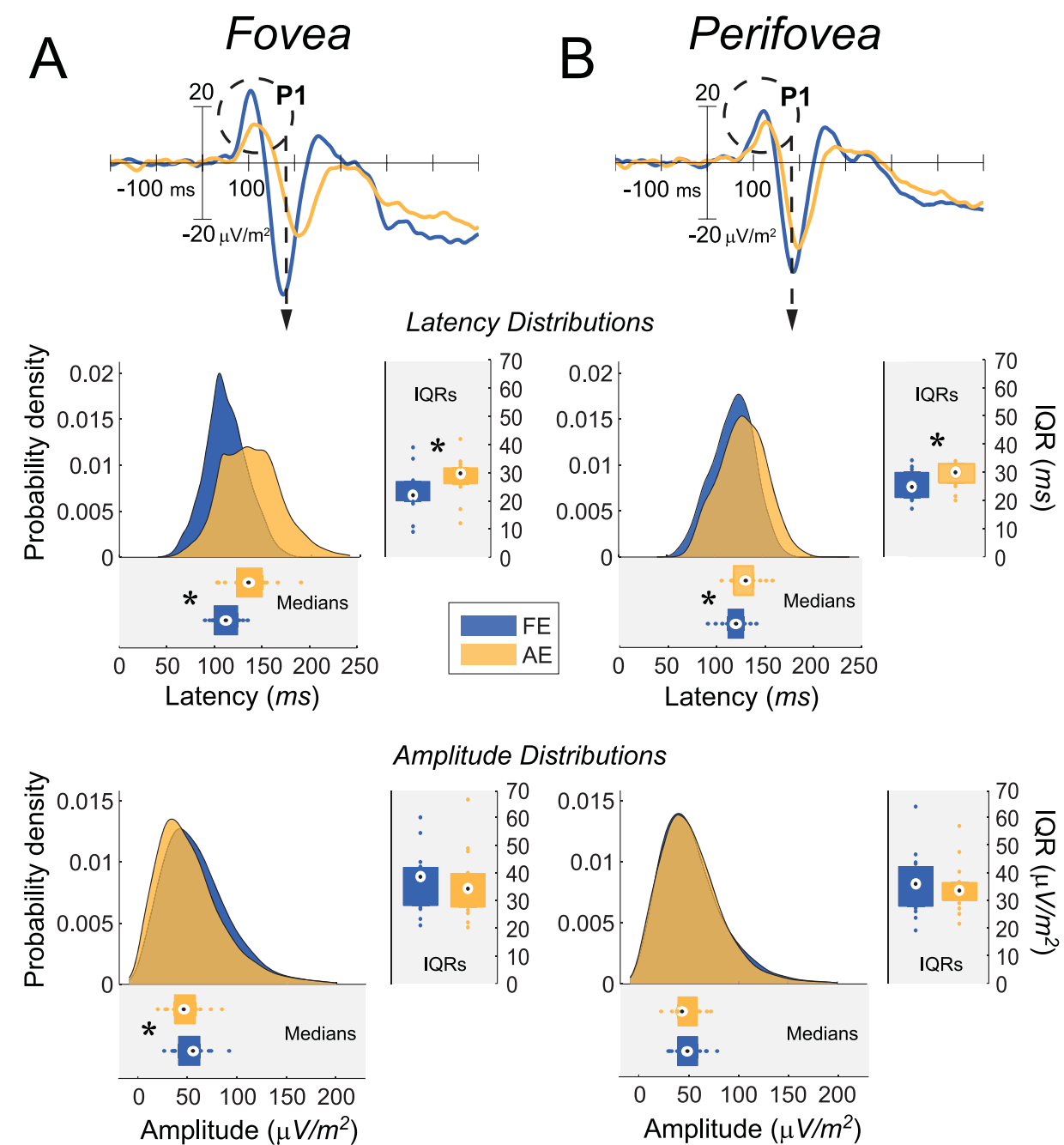

Amplitude Distributions

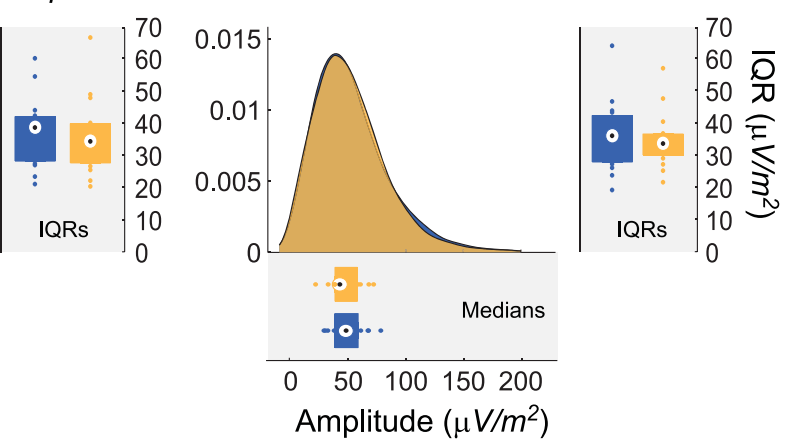

Figune 3. P1 amplitude and latency distributions obtained over the right hemisphere in the case of foveal (A) and perifoveal (B) stimuli, which were matched in size according to the cortical magnification factor. Top: averaged ERPs from the right electrode cluster (P8, P10, PO8, and PO10); middle and bottom: probability density functions (pdf) of latency and amplitude distributions of the two eyes, respectively. The pdfs were estimated individually using a normal kernel function, averaged across subjects, and serve visualization purposes only. Individual parameters of the distributions (colored dots) are plotted below (medians) and to the right (IQRs) of each distribution panel, where the black dot and the box indicate the median and the $25 \%$ to $75 \%$ range (IQR) of the data sets, respectively $(n=15$; asterisks denote significant interocular differences: $P<0.013$; negative is down for the ERP traces).

\section{Discussion}

We have shown that amblyopic deficits exist in the ERP responses recorded outside the central visual field. These can be reliably detected when the size of the peripheral stimulus corresponds to the size of the fovea scaled by cortical magnification. Stimulating a much larger cortical area, on the other hand, may render the deficit statistically unnoticeable. Our results have revealed for the first time that foveal and peripheral deficits differ in nature. Deficit at the fovea arises as a mixture of decreased single-trial amplitude and delayed, uncertain timing of the ERP responses. Conversely, the amblyopic deficit outside the fovea is dominantly characterized by a deficiency in timing of neural responses, while the contribution of response magnitude reduction to the observed effects is negligible.

\section{Importance of Cortical Magnification}

Our results, demonstrating that the sensitivity of the ERPs for detecting amblyopic effects at the perifovea might depend on adequate stimulus scaling, may help to reconcile the divergent VEP results concerning the presence or absence of the amblyopic deficit outside the fovea. ${ }^{11,13-15,17-19}$ They are also in agreement with previous observations that magnification scaling is a highly important variable that influences the elicited pattern evoked potential. ${ }^{13}$ Our findings also closely parallel the psychophysical results of Katz and colleagues, ${ }^{2}$ who showed that peak contrast sensitivity of amblyopic eyes at the periphery benefitted more from an increase in stimulus size, reaching the sensitivity of the fellow eyes for large central stimuli. They concluded that spatial summation across the extent of the stimulus field increased peak contrast sensitivity at least for the amblyopic eye. ${ }^{2}$

\section{Deficient ERP Response Timing in Amblyopia}

The amblyopic deficit in timing of the neural responses is similar across the visual field apart from the fact that the interocular difference at the perifovea appears to be attenuated compared with the difference at the fovea for both latency delay and jitter. Neurophysiological research on strabismic cats 


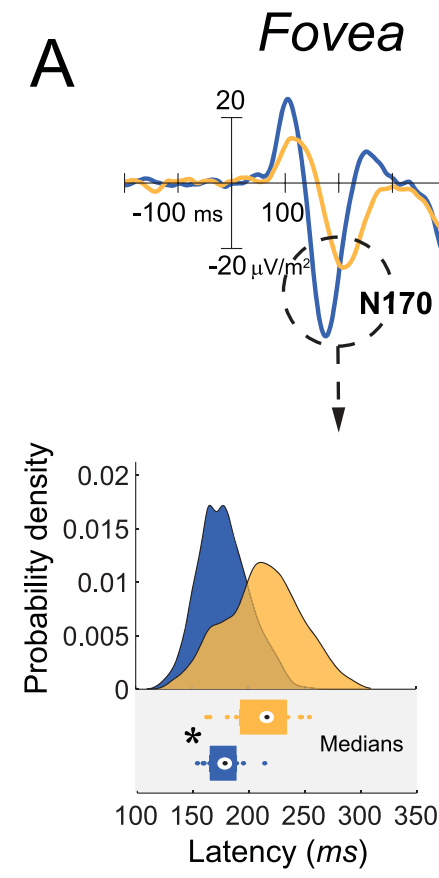

\section{B Perifovea}

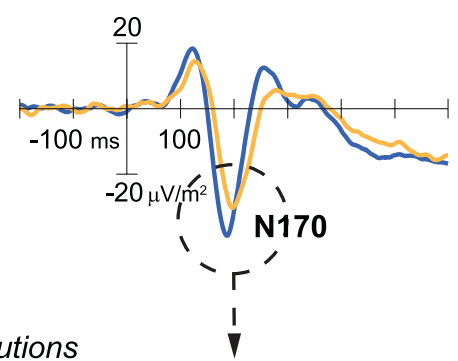

Latency Distributions

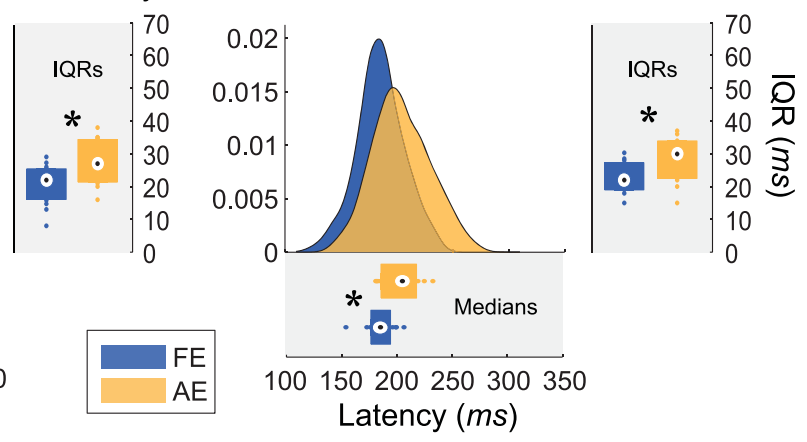

Amplitude Distributions
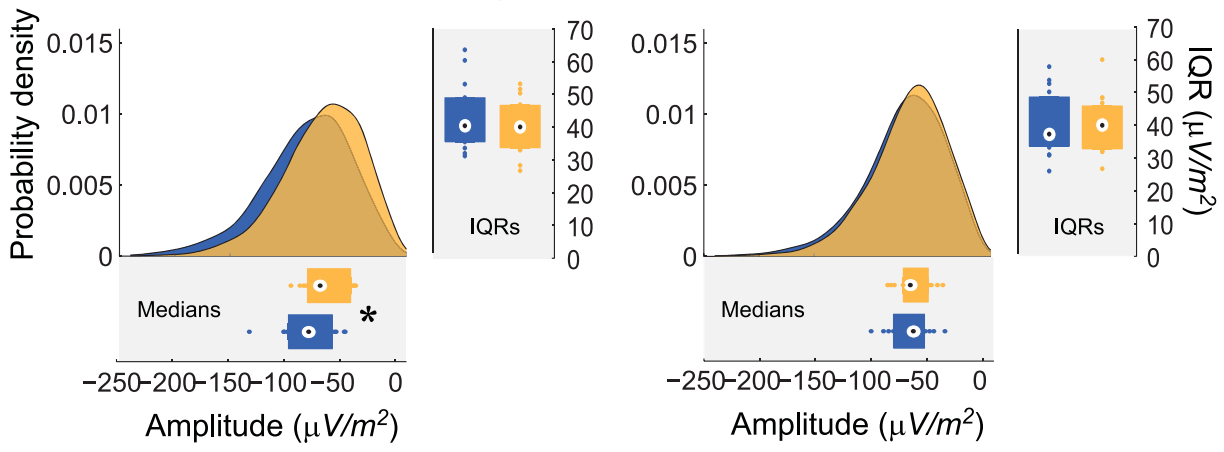

Figure 4. N170 amplitude and latency distributions obtained over the right hemisphere in the case of foveal (A) and perifoveal (B) stimuli, which were matched in size according to the cortical magnification factor. Top: averaged ERPs from the right electrode cluster (P8, P10, PO8, and PO10); middle and bottom: pdfs of latency and amplitude distributions of the two eyes, respectively. The pdfs were estimated individually using a normal kernel function, averaged across subjects, and serve visualization purposes only. Individual parameters of the distributions (colored dots) are plotted below (medians) and to the right (IQRs) of each distribution panel, where the black dot and the box indicate the median and the $25 \%$ to $75 \%$ range (IQR) of the data sets, respectively ( $n=15$; asterisks denote significant interocular differences: $P<0.013$; negative is down for the ERP traces).

has revealed that neuronal response latencies in primary visual cortical neurons driven by the amblyopic eye are also delayed $^{35,36}$ and highly variable, as reflected by decreased neural synchrony, ${ }^{37}$ compared with visual neurons driven by the fellow eye. The increased variance in the timing of neural activity represents an increase in internal neural noise, which comprises random internal noise-a crucial factor in many models used to explain the psychophysical performance of the amblyopic eye. ${ }^{38-41}$ This ties in with our results of greater trialto-trial variability of ERP component latency coming from the amblyopic eye, implying that an overall uncertainty in the timing of neural responses might underlie the increase in internal noise observed in amblyopia. Similar timing deficiencies were found by Milne ${ }^{42}$ in autism spectrum disorder (ASD); the trial-to-trial variability (i.e., jitter) of P1 latency was found to be significantly higher than that of the matched control group, suggesting that individuals with ASD are less able to synchronize the activity of stimulus-related cell assemblies and display increased neural noise compared with healthy controls.

\section{Unaltered Amblyopic ERP Response Strength at the Periphery}

In the ERPs obtained with perifoveal stimulation, we found very weak, nonsignificant interocular changes in the single-trial amplitude as compared with the strong reduction at the fovea. At least two phenomena could possibly account for this. First, it has been shown that induced refractive error causes amplitude reduction of VEP components, which is most pronounced for stimuli with higher spatial frequency (e.g., checks of $5^{\prime}-40^{\prime}$ of arc). ${ }^{20,21}$ Since our sensitivity for high spatial frequencies decreases toward the periphery of the visual field, stimulation farther away from the fovea becomes less susceptible to the effects of degraded visual acuity. Accordingly, the acuity deficit of the amblyopic eye also lessens toward the periphery. ${ }^{5-7}$ Our finding that the removal of higher spatial frequency content from the stimuli reduced the amplitude of the P1 component only at the fovea but not at the periphery is also in agreement with the above. Thus, the degraded visual acuity of the amblyopic eye could have 


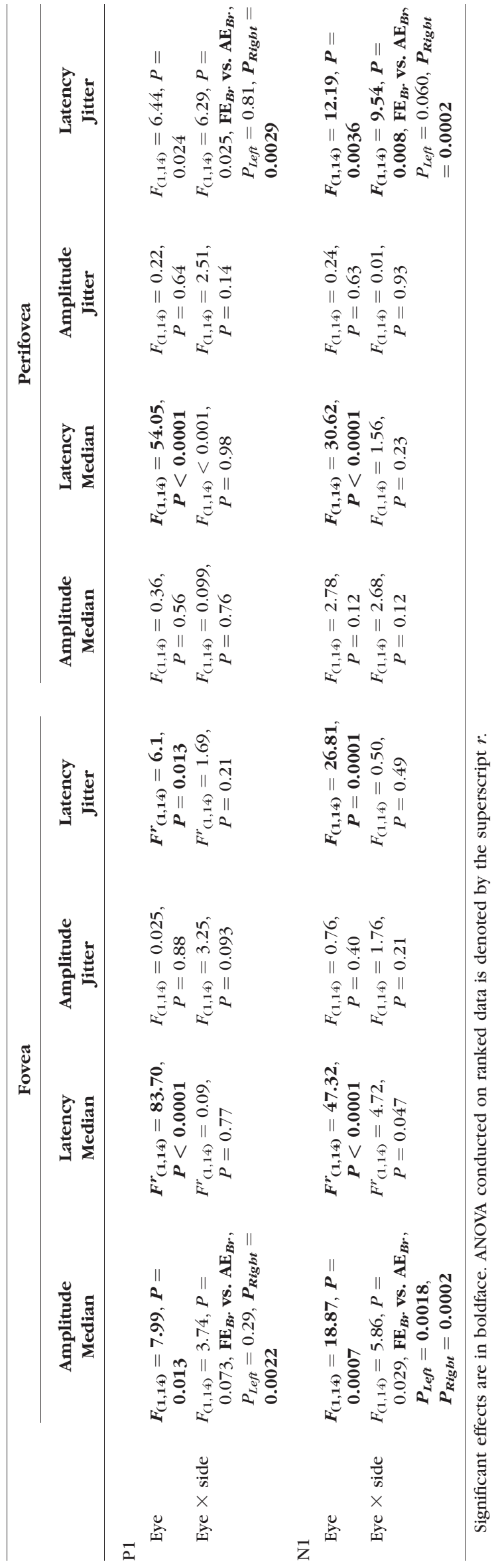

contributed to the amblyopic amplitude reduction under foveal viewing in the case of P1 while it did not affect single-trial amplitudes at the perifovea. Nevertheless, it is important to note that the amblyopic effect on the averaged ERPs was present for low-pass-filtered stimuli, indicating that it is not simply the result of the inability of the amblyopic eye to perceive high spatial frequencies. Secondly, unsteady fixation, a known problem for amblyopic patients, ${ }^{19,27,29-31}$ can also lead to reductions in the observed amplitude. Artificially induced fixation errors greatly affect VEP waveforms especially at the fovea, but the effects have been found to be minimal outside the central $5^{\circ}$ to $6^{\circ}$ of the visual field in the case of approximately $1^{\circ}$ fixation error $^{19}$ (Menz M, et al. IOVS 2002; 43:ARVO E-Abstract 4740). Thus, unsteady fixation is likely to contribute to amblyopic averaged amplitude reduction at the fovea. Nevertheless, it is unclear whether fixation instability affects true evoked potential magnitude or increases the trial-to-trial latency variability of the responses. To elucidate this, further studies using induced fixation instability are needed.

\section{Conclusions}

In conclusion, our findings suggest that the amblyopic deficit observed in evoked responses outside the fovea can mainly be regarded as a timing deficit, while at the fovea it is a combination of decreased response strength and faulty timing. This overall uncertainty in response timing might form the neural basis for increased internal noise. In addition, our results emphasize the importance of controlling for cortical magnification when evaluating amblyopic vision in the periphery.

\section{Acknowledgments}

Supported by a grant from the Hungarian Scientific Research Fund (OTKA CNK 80369) to ZV and by the János Bolyai Research Scholarship of the Hungarian Academy of Sciences (ÉMB).

Disclosure: É.M. Bankó, None; J. Körtvélyes, None; J. Németh, None; Z. Vidnyánszky, None

\section{References}

1. Burian HM. The behavior of the amblyopic eye under reduced illumination and the theory of functional amblyopia. Doc Ophthalmol. 1967;23:189-202.

2. Katz LM, Levi DM, Bedell HE. Central and peripheral contrast sensitivity in amblyopia with varying field size. Doc Ophthalmol. 1984;58:351-373.

3. Levi DM, Klein SA, Aitsebaomo P. Detection and discrimination of the direction of motion in central and peripheral vision of normal and amblyopic observers. Vision Res. 1984;24:789800.

4. Hess RF, Pointer JS. Differences in the neural basis of human amblyopia: the distribution of the anomaly across the visual field. Vision Res. 1985;25:1577-1594.

5. Kirschen DG, Flom MC. Visual acuity at different retinal loci of eccentrically fixating functional amblyopes. Am J Optom Pbysiol Opt. 1978;55:144-150.

6. Hess RF, Jacobs RJ. A preliminary report of acuity and contour interactions across the amblyope's visual field. Vision Res. 1979;19:1403-1408.

7. Sireteanu R, Fronius M. Naso-temporal asymmetries in human amblyopia: consequence of long-term interocular suppression. Vision Res. 1981;21:1055-1063.

8. Sireteanu R, Fronius M, Singer W. Binocular interaction in the peripheral visual field of humans with strabismic and anisometropic amblyopia. Vision Res. 1981;21:1065-1074. 
9. Kiorpes L, Kiper DC, O'Keefe LP, Cavanaugh JR, Movshon JA. Neuronal correlates of amblyopia in the visual cortex of macaque monkeys with experimental strabismus and anisometropia. J Neurosci. 1998;18:6411-6424.

10. Lerner Y, Hendler T, Malach R, et al. Selective fovea-related deprived activation in retinotopic and high-order visual cortex of human amblyopes. Neuroimage. 2006;33:169-179.

11. Parisi V, Scarale ME, Balducci N, Fresina M, Campos EC. Electrophysiological detection of delayed postretinal neural conduction in human amblyopia. Invest Ophthalmol Vis Sci. 2010;51:5041-5048.

12. Sokol S. Abnormal evoked potential latencies in amblyopia. $\mathrm{Br}$ J Ophthalmol. 1983;67:310-314.

13. Meredith JT, Celesia GG. Pattern-reversal visual evoked potentials and retinal eccentricity. Electroencephalogr Clin Neurophysiol. 1982;53:243-253.

14. Odom JV, Bach M, Brigell M, et al. ISCEV standard for clinical visual evoked potentials (2009 update). Doc Ophthalmol. 2010;120:111-119.

15. Harter MR. Evoked cortical responses to checkerboard patterns: effect of check-size as a function of retinal eccentricity. Vision Res. 1970;10:1365-1376.

16. Levi DM, Walters JW. Visual evoked responses in strabismic and anisometropic amblyopia: effects of check size and retinal locus. Am J Optom Physiol Opt. 1977;54:691-698.

17. Moschos M, Margetis I, Tsapakis S, Panagakis G, Chatzistephanou I, Iliakis E. Multifocal visual evoked potentials in amblyopia due to anisometropia. Clin Ophthalmol. 2010;4: 849-853.

18. Yu M, Brown B, Edwards MH. Investigation of multifocal visual evoked potential in anisometropic and esotropic amblyopes. Invest Ophthalmol Vis Sci. 1998;39:2033-2040.

19. Zhang B, Stevenson SS, Cheng H, et al. Effects of fixation instability on multifocal VEP (mfVEP) responses in amblyopes. J Vis. 2008;8:16.1-16.14.

20. Harter MR, White CT. Evoked cortical responses to checkerboard patterns: effect of check-size as a function of visual acuity. Electroencephalogr Clin Neurophysiol. 1970;28:4854.

21. Harter MR, White CT. Effects of contour sharpness and checksize on visually evoked cortical potentials. Vision Res. 1968;8: 701-711.

22. Baseler HA, Sutter EE, Klein SA, Carney T. The topography of visual evoked response properties across the visual field. Electroencephalogr Clin Neurophysiol. 1994;90:65-81.

23. Horton JC, Hoyt WF. The representation of the visual field in human striate cortex. A revision of the classic Holmes map. Arch Ophthalmol. 1991;109:816-824.

24. Hood DC, Odel JG, Winn BJ. The multifocal visual evoked potential. J Neuroophthalmol. 2003;23:279-289.

25. Abramoff MD, Magalhães PJ, Ram SJ. Image processing with ImageJ. Biophotonics Intern. 2004;11:36-42.
26. Lerner Y, Pianka P, Azmon B, et al. Area-specific amblyopic effects in human occipitotemporal object representations. Neuron. 2003;40:1023-1029.

27. Bankó ÉM, Körtvélyes J, Németh J, Weiss B, Vidnyánszky Z. Amblyopic deficits in the timing and strength of visual cortical responses to faces. Cortex. 2013;49:1013-1024.

28. Dougherty RF, Koch VM, Brewer AA, Fischer B, Modersitzki J, Wandell BA. Visual field representations and locations of visual areas V1/2/3 in human visual cortex. J Vis. 2003;3:586-598.

29. Schor CM, Westall C. Visual and vestibular sources of fixation instability in amblyopia. Invest Ophthalmol Vis Sci. 1984;25: 729-738.

30. Bedell HE, Yap YL, Flom MC. Fixational drift and nasaltemporal pursuit asymmetries in strabismic amblyopes. Invest Ophthalmol Vis Sci. 1990;31:968-976.

31. González EG, Wong AMF, Niechwiej-Szwedo E, Tarita-Nistor L, Steinbach MJ. Eye position stability in amblyopia and in normal binocular vision. Invest Ophthalmol Vis Sci. 2012;53:53865394.

32. Levi DM, Manny RE. The pathophysiology of amblyopia: electrophysiological studies. Ann N YAcad Sci. 1980;338:243260.

33. Anderson SJ, Holliday IE, Harding GF. Assessment of cortical dysfunction in human strabismic amblyopia using magnetoencephalography (MEG). Vision Res. 1999;39:1723-1738.

34. Davis AR, Sloper JJ, Neveu MM, Hogg CR, Morgan MJ, Holder GE. Electrophysiological and psychophysical differences between early- and late-onset strabismic amblyopia. Invest Ophthalmol Vis Sci. 2003;44:610-617.

35. Singer W, von Grünau M, Rauschecker J. Functional amblyopia in kittens with unilateral exotropia. I. Electrophysiological assessment. Exp Brain Res. 1980;40:294-304.

36. Eschweiler GW, Rauschecker JP. Temporal integration in visual cortex of cats with surgically induced strabismus. Eur $J$ Neurosci. 1993;5:1501-1509.

37. Roelfsema PR, König P, Engel AK, Sireteanu R, Singer W. Reduced synchronization in the visual cortex of cats with strabismic amblyopia. Eur J Neurosci. 1994;6:1645-1655.

38. Levi DM, Klein SA. Noise provides some new signals about the spatial vision of amblyopes. $J$ Neurosci. 2003;23:2522-2526.

39. Levi DM, Klein SA, Chen I. The response of the amblyopic visual system to noise. Vision Res. 2007;47:2531-2542.

40. Huang C, Tao L, Zhou Y, Lu Z-L. Treated amblyopes remain deficient in spatial vision: a contrast sensitivity and external noise study. Vision Res. 2007;47:22-34.

41. Baker DH, Meese TS, Hess RF. Contrast masking in strabismic amblyopia: attenuation, noise, interocular suppression and binocular summation. Vision Res. 2008;48:1625-1640.

42. Milne E. Increased intra-participant variability in children with autistic spectrum disorders: evidence from single-trial analysis of evoked EEG. Front Psychol. 2011;2:51. 